\title{
Optimizing Communication in Air-Ground Robot Networks using Decentralized Control
}

\author{
Stephanie Gil, ${ }^{*}$ Mac Schwager, ${ }^{*}$ Brian J. Julian, ${ }^{* \dagger}$ and Daniela Rus ${ }^{\ddagger}$ \\ ${ }^{*}$ Computer Science and Artificial Intelligence Lab (CSAIL), MIT, Cambridge, MA 02139, USA \\ ${ }^{\dagger}$ MIT Lincoln Laboratory, 244 Wood Street, Lexington, MA, 02420, USA \\ Email: sgil@mit.edu,schwager@mit.edu, bjulian@mit.edu, and rus@csail.mit.edu
}

\begin{abstract}
We develop a distributed controller to position a team of aerial vehicles in a configuration that optimizes communication-link quality, to support a team of ground vehicles performing a collaborative task. We propose a gradientbased control approach where agents' positions locally minimize a physically motivated cost function. The contributions of this paper are threefold. We formulate of a cost function that incorporates a continuous, physical model of signal quality, SIR. We develop a non-smooth gradient-based controller that positions aerial vehicles to acheive optimized signal quality amongst all vehicles in the system. This controller is provably convergent while allowing for non-differentiability due to agents moving in or out of communication with one another. Lastly, we guarantee that given certain initial conditions or certain values of the control parameters, aerial vehicles will never disconnect the connectivity graph. We demonstrate our controller on hardware experiments using AscTec Hummingbird quadrotors and provide aggregate results over 10 trials. We also provide hardware-in-the-loop and MATALB simulation results, which demonstrate positioning of the aerial vehicles to minimize the cost function $H$ and improve signal-quality amongst all communication links in the ground/air robot team.
\end{abstract}

\section{INTRODUCTION}

Distributed control of groups of robots working collaboratively to acheive a task has been the focus of many recent research efforts. These systems are particularly interesting because of their inherent robustness to failures, and because of their potential to solve a large range of interesting problems such as the exploration of an environment, search and rescue tasks, collaborative construction, and the modeling of biological systems. However, many applications of distributed systems require that agents work at large distances from one another, or in noisy environments, where communication quality can degrade or be lost altogether. The National Aeronautics and Space Administration (NASA) has recently focused attention on swarm-based missions where hundreds or even thousands of intelligent spacecraft will work in teams to achieve collaborative tasks in space exploration [1]. The

This work was done in the Distributed Robotics Laboratory at MIT. This work was supported and in part by the ARO MURI SWARMS grant 544252, the ARL CTA MAST grant 549969, the ONR MURI SMARTS grant N0014-09-1051, NSF grants IIS-0513755, IIS-0426838, EFRI-0735953, Bell Labs Graduate Fellowship, MIT Lincoln Laboratory, and The Boeing Company.

This work is sponsored by the Department of the Air Force under Air Force contract number FA8721-05-C-0002. The opinions, interpretations, recommendations, and conclusions are those of the authors and are not necessarily endorsed by the United States Government. case of exploration in an unknown environment with ambient noise exemplifies the need for communication networks that can be optimized adaptively. We propose a nonsmooth, gradient-based approach to positioning a group of aerial vehicles in a configuration that optimizes communicationlink quality amongst a team of ground vehicles performing an independent, collaborative task. We acheive this objective via careful design of an appropriate cost function that is then minimized by the placement of the aerial vehicles.

A common approach to distributed minimization of a cost function is to design a gradient-based controller where agents follow a distributed gradient descent on that cost function. We design a cost function that incorportates the Signal-ToInterference Ratio (SIR) from the communication literature, which is a physically-based, continuous measure of link quality between any two communicating agents [2]. Local minima of our cost function achieve a tradeoff between maximizing the SIR for any single link, and equalizing the communication capability, also SIR, over all links in the graph. We model signal strength between two agents that degrades with distance and drops non-smoothly to zero outside of the communication radius $R$. The non-differentiability due to agents entering or leaving the communication radius of one another necessitates the use of results from the nonsmooth stability analysis literature [3] to prove convergence to local minima of the cost function. Furthermore, for certain initial conditions and controller parameter values, we prove that aerial vehicles will never move in such a way so as to disconnect the communication graph.

We implement our controller on a team of AscTec Hummingbird flying quadrotor robots providing network coverage for ground vehicles, using xBee-PRO modules for wireless communication. We present aggregate results of ten hardware experiment trials, demonstrating positioning of a team of three quadrotor aerial vehicles to provide optimized communication for a group of three ground vehicles. We also present the results of hardware-in-the-loop simulations for up to three aerial vehicles and four ground vehicles, and MATLAB simulation results for up to eight aerial vehicles and eight ground vehicles. Our MATLAB simulations also show that we can adjust the behavior of the aerial vehicles to optimize SIR values over individual links, or an equalization of SIR values over all links in the communication graph, by adjusting a design parameter $\lambda$ in the cost function $H$. 


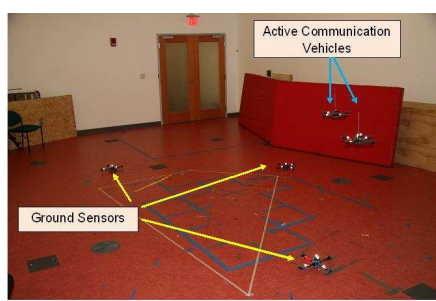

(a)

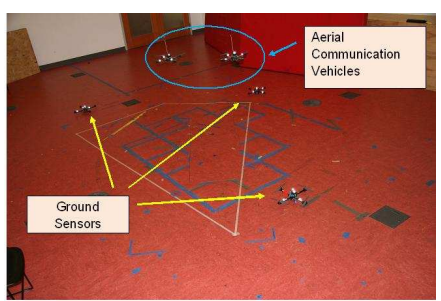

(b)

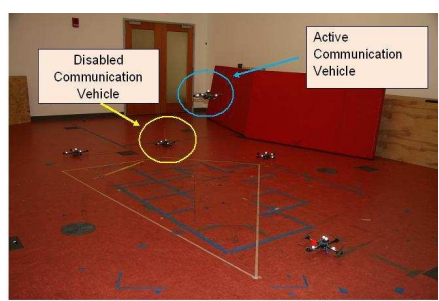

(c)

Fig. 1. These figures show the initial and converged configurations for two aerial vehicles and three ground sensors. Figure 1(c) demonstrates the new equilibrium acheived when one flier is re-assigned to a ground station.

\section{A. Related Work}

The development of distributed control of groups of robots working collaboratively to achieve a task has been a research focus in broad ranging fields including dynamic routing problems [4], [5], collaborative construction tasks [6], modeling of biological systems, and coverage [7], [8]. In many of these applications communication across the network is an important and challenging problem. The paper [9] concerns formation control of agents under communication constraints. Other work concerns using a communication tether to link a ground, or base station, to an exploring agent [10], [11]. The paper [12] addresses the communication problem by integrating information theoretic measures into the objective function and demonstrates this approach on a chain configuration of mobile robots.

A second challenge we address in this paper is to ensure that aerial vehicles will never move to disconnect the communication graph. This is a difficult problem in a distributed system because each agent's controller only accounts for local information and the connectivity status is a global property of that graph. Other research efforts have focused attention solely on the problem of maintaining connectivity for distributed systems [13], [10], [14]. Many of these works use distributed algorithmic methods of checking the connectivity of the graph via gossip algorithms, local minimum spanning trees, or other iterative approaches. Our approach allows for a continuous method of connectivity maintenence using local information at the expense of a more conservative controller. Less conservative approaches to this problem could involve a combination of our distributed controller for communication optimization and an algorithmic check for graph connectivity such as the work in [14].

This paper is organized as follows: Section II describes the problem and our approach, Section III provides the nonsmooth convergence analysis of our controller and proof of connectivity maintenence, and Section IV presents the experimental and MATLAB results. We conclude with Section V.

\section{PROBLEM Formulation}

We are interested in the problem where $n$ ground vehicles, performing a collaborative task such as coverage, search, or exploration of an environment, are required to communicate over distances greater than their communication radius $R$ in order to acheive their assigned task. We propose the use of a group of $m$ aerial vehicles to provide a communication network for the ground vehicles, where the aerial robots follow a distributed control law and are placed at locations that optimize communication link quality amongst all vehicles according to a specific cost $H$. We assume that 1) $m$ is large enough to provide a connected network amongst ground vehicles, 2) that communication only exists amongst neighbors within a distance radius $R$ where signal strength is modeled by $f_{i j}$ described later in this section, and that 3 ) the ground vehicle dynamics are zero as necessary for the mathematical proof, although in the practical setting we may allow ground vehicles to move given that their velocities are much smaller than those of the aerial vehicles. We note that assumption 3 is common for problems using Lyapunov-type proofs of stability. Due to the distributed nature of our problem, all agents have access only to local information and thus will be unaware of disconnected subclusters. Therefore we must also assume that the communication network composed of both air and ground vehicles is initially in a connected state, although our controller is robust to changes in the network including agents arriving or exiting. Our hardware results demonstrated in Figure 4 include such a scenario, where an aerial vehicle is disabled and the remaining aerial vehicle positions themselves to compensate for the loss of the aerial vehicle.

We aim to ensure connectivity of the graph in a continuous fashion by either placing a requirement that the initial conditions of the system are below some critical cost, or by adjusting a design parameter $\lambda$ in our cost function to ensure that aerial vehicles will never break existing connections.

Aerial vehicles are controlled via a gradient descent method, where we allow for a nonsmooth cost function that is non-differentiable at the points where agents come in and out of communication radius of each other. Due to the local non-differentiability of the cost function, we must instead use the generalized gradient of the cost function which we denote $\frac{\partial H}{\partial x_{i}}$ throughout. We find the direction of descent for the resulting nonsmooth gradient vector field such that the controller takes the form

$$
\dot{x_{i}}=-\operatorname{Ln}(\partial H)\left(x_{i}\right) .
$$

Where $\operatorname{Ln}(\partial H)(x i): \mathbb{R}^{d} \rightarrow \mathbb{R}^{d}$ is the generalized gradient vector field, and $-\operatorname{Ln}(\partial H)\left(x_{i}\right)$ is a direction of descent of 
$H$ at $x_{i} \in \mathbb{R}^{d}$ [3]. In Section III we find the generalized gradient vector field of the cost function and show that the resulting positions of the aerial vehicles converge to critical points of this cost function.

We design our cost function to incorporate a physicallybased, continuous, measure of signal quality called the Signal-to-Intereference Ratio (SIR) [2]. The SIR value of the link $i-j$ improves with increasing communication strength between agents $i$ and $j$ and decreases with increasing environmental noise $N_{i}$ and interfering communication amongst $i$ 's other neighbors as seen from the definition of SIR:

$$
S I R_{i j}=\frac{f_{i j}}{N_{i}+\sum_{k \in \mathcal{N}_{i \backslash j}} f_{i k}}
$$

Where $\mathcal{N}_{i \backslash j}$ is the set of neighbors of $i$ not including $j$. The communication strength over link $i-j$ is denoted $f_{i j}$. We choose an example model for the signal strength that drops off proportional to $d_{i j}{ }^{-\alpha}$, but we emphasize that other, more problem specific models for signal strength can be used with our controller so long as this function is locally Lipschitz and regular and models no communication outside of the radius $R$. These properties are important for the analysis of our controller but we defer this discussion to section III. We define $f_{i j}$ as

$$
f_{i j}=\left\{\begin{aligned}
\frac{P_{0}}{d_{i j}^{\alpha}+1}-C & , \quad d_{i j} \leq R \\
0 & , \quad d_{i j} \geq R
\end{aligned}\right.
$$

where $C=\frac{P_{0}}{R^{\alpha}}$ is a constant to ensure continuity at $d_{i j}=R$, and we define $d_{i j}=\|i-j\|$. Thus the communication strength model reaches a maximum value of $\frac{P_{0}}{d_{i j}^{\alpha}+1}-C$ at $d_{i j}=0$ and drops off by $\alpha$ as $d_{i j}>0$ with a nonsmooth transition to zero at $d_{i j}=R$ as seen in Figure 2 . This non-smooth transition is necessary to model loss of communication between two agents at a distance larger than $R$ from each other. Finally, we present our cost function $H$.

$$
H=\sum_{i} \sum_{j \neq i}-S I R_{i j}+\frac{\lambda}{S I R_{i j}+\delta}
$$

Where the term $\delta \in(0,1]$ is included to ensure that the cost function $H$ is continuous at the point where agents become disconnected and the value of $S I R_{i j}=0$. A smaller $\delta$ value has the effect of putting more weight on the second term of the cost function. It is evident that the cost function is global and thus uses position information for all agents. However, as shown in equation (8) the control for each agent is local, as all non-neighbor information drops out in the derivative. Figure 6 shows optimization of a non-smooth $\mathrm{H}$ as agents enter the communication neighborhoods of others.

Minimization of this cost function corresponds to a compromise of two competing goals. The first term in the cost function favors increased SIR over all communication links in the graph while the second term favors equal SIR over each individual link, which can be thought of as equal resource allocation where SIR measures communication ability

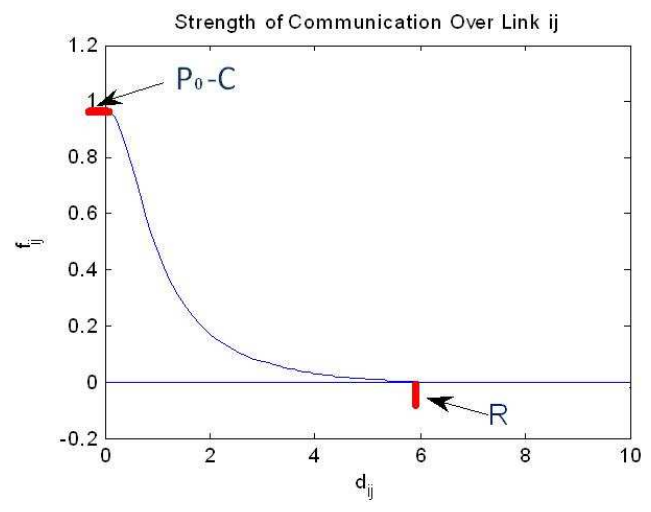

Fig. 2. Plot of $f_{i j}$.

of each link. The design parameter $\lambda$ is used to adjust the weighting of the first term versus the second term in the cost function. A higher weighting on the second term corresponds to agents seeking to equalize their SIR values amongst all of their neighbors whereas a higher weighting on the first term will result in agents greedily improving individual SIR links. In Section III we prove that there exists a critical value of $\lambda, \lambda_{\mathrm{cr}}$, that prevents agents from disconnecting from existing neighbors and demonstrate this range of behaviors for the controller in Figure 3.

Because the cost function $H$ is non-smooth due to the nondifferentiability of $f_{i j}$ at $d_{i j}=R$, our controller requires a non-smooth stability analysis as described in the next section.

\section{NON-SMOOTH ANALYSIS}

In this section of the paper we present the stability analysis of the controller presented in (1). We also describe the sufficient conditions to ensure connectivity preservation for the communication graph.

\section{A. Non-Smooth Analysis of Controller}

The cost function $H$ presented in Section II is nonsmooth at the point where agents move in and out of the communication radius $R$ of each other. This is reflected as a non-smooth transition to zero in the function $f_{i j}$ at the point $d_{i j}=R$. As a result, the derivative does not exist at this point and we must instead find the generalized gradient and generalized gradient vector field of our cost function in order to build the appropriate controller.

1) Generalized Gradient and the Generalized Gradient Vector Field: Following the theory of discontinuous dynamical systems, due to the local non-differentiability of $H$, the controller in (1) in fact uses the generalized gradient $\frac{\partial H}{\partial x_{i}}$. The generalized gradient of a function $f$ at a point of nondifferentiability, $x$, is presented in [3], as the convex hull of the all the possible limits of the gradient at neighboring points where the gradient is defined. More precisely:

$$
\frac{\partial H}{\partial x}=c o\left\{\lim _{z_{i} \rightarrow z} \nabla H\left(z_{i}\right) \forall z_{i}: z_{i} \rightarrow z, z_{i} \notin \Omega_{H}\right\} .
$$


where co denotes convex hull, $H: \mathbb{R}^{d} \rightarrow \mathbb{R}$ is a locally Lipschitz function, and $\Omega_{H} \subset \mathbb{R}^{d}$ denotes the set of points where $H$ fails to be differentiable. Moreover, the generalized gradient vector field, $\operatorname{Ln}\left(\frac{\partial H}{\partial x}\right): \mathbb{R}^{d} \rightarrow \mathbb{R}^{d}$, is defined in [3] where $L n: \mathcal{B}\left(\mathbb{R}^{d}\right) \rightarrow \mathcal{B}\left(\mathbb{R}^{d}\right)$ is a set-valued map that associates to each subset $\mathcal{S}$ of $\mathbb{R}^{d}$ the set of least-norm elements of its closure $\overline{\mathcal{S}}$. Most importantly, $-\operatorname{Ln}\left(\frac{\partial H}{\partial x}\right)$ is a direction of descent of $H$ at $x \in \mathbb{R}^{d}$ [3]. Finding the generalized gradient for an arbitrary nonsmooth function can be a daunting task, however for our case, because the function $f_{i j}$ is smooth everywhere except at $R$, the generalized gradient is equivalent to the normal gradient at all points outside of $R$, where at $R$ it takes the value zero. The generalized gradient vector field of $f_{i j}$ for our problem is:

$$
\begin{aligned}
& \operatorname{Ln}\left[\frac{\partial f_{i j}}{\partial x_{i}}\right]= \\
& \left\{\begin{array}{rr}
\left\{-\frac{\alpha P\left(x_{i}-x_{j}\right)\left\|x_{i}-x_{j}\right\|^{\alpha-2}}{\left(\left\|x_{i}-x_{j}\right\|^{\alpha}+1\right)^{2}}\right\} & , \quad d_{i j}<R \\
\{0\} & , \quad d_{i j} \geq R
\end{array}\right.
\end{aligned}
$$

Knowing the generalized vector field for $f_{i j}$ is sufficient for finding the generalized vector field of the cost function $H$. This relies on the fact that $f_{i j}$ is Lipschitz and regular. A function is said to be locally Lipschitz at $x \in \mathbb{R}^{d}$ if there exist a $L_{x}$ and $\epsilon \in(0, \infty)$ such that $\left\|f(y)-f\left(y^{\prime}\right)\right\| \leq$ $L_{x}\left\|y-y^{\prime}\right\|$ for all $y, y^{\prime} \in B(x, \epsilon)$ where $B(x, \epsilon)$ is a ball centered at $x$ of radius $\epsilon$. A function is said to be regular when its right directional derivative $f^{\prime}(x ; v)$ is equal to its generalized directional derivative $f^{0}(x ; v)$, [3], where:

$$
f^{0}(x ; v)=\lim _{h \rightarrow 0^{+}} \sup _{y \rightarrow x} \frac{f(y+h v)-f(y)}{h}
$$

The proof of $f_{i j}$ Lipschitz and regular, as well as the final form of the controller using the generalized vector field of $H$ is presented in the next subsection.

2) Stability of Controller: We present our main stability result as Proposition 1 but we first present supporting results from the nonsmooth analysis literature. The first results are the Sum Rule and Quotient Rule for algebraic operations on nonsmooth functions summarized in [3]. These results are important for conserving Lipschitz and regular properties of nonsmooth functions and for finding the generalized gradient of a function that is an algebraic composition of such functions.

Sum Rule: If $f_{1}, f_{2}: \mathbb{R}^{d} \rightarrow \mathbb{R}$ are locally Lipschitz and regular at $x \in \mathbb{R}^{d}$ and $s_{1}, s_{2} \in \mathbb{R}$, then the function $s_{1} f_{1}+s_{2} f_{2}$ is locally Lipschitz and regular at $x$ and the generalized gradient $\partial\left(s_{1} f_{1}+s_{2} f_{2}\right)(x)=s_{1} \partial f_{1}+s_{2} \partial f_{2}$.

Quotient Rule: If $f_{1}, f_{2}: \mathbb{R}^{d} \rightarrow \mathbb{R}$ are locally Lipschitz and regular at $x \in \mathbb{R}^{d}$ and $s_{1}, s_{2} \in \mathbb{R}$, then the function $f_{1} / f_{2}$ is locally Lipschitz and regular at $x$ and the generalized gradient $\partial\left(f_{1} / f_{2}\right)(x)=\left(1 / f_{2}^{2}(x)\right)\left(f_{2} \partial f_{1}-f_{1} \partial f_{2}\right)$.

We combine the results Theorem 1 and Theorem 2 of Jorge Cortes' Discontinuous Dynamical Systems to produce a result similar to Proposition 11 of the same work. We state this result here as Lemma 1.

Lemma 1: Let $H: \mathbb{R}^{d} \rightarrow \mathbb{R}$ be locally Lipschitz and regular. Then, the strict minimizers of $H$ are strongly stable equilibria of the nonsmooth gradient flow of $H$. Furthermore, if there exists a compact and strongly invariant set for the nonsmooth dynamics in (1), then the solutions of the nonsmooth gradient flow asymptotically converge to the set of critical points of $H$ [3].

We are now ready to state and prove our theorem for stability and convergence properties of our controller in (1).

Theorem 1: Aerial vehicles following the direction of descent of the generalized gradient of $H$ such that $\dot{x}_{i}(t)=-\operatorname{Ln}\left(\frac{\partial H}{\partial x_{i}}\right)$ will asymptotically converge to the critical points of $H$ where the strongly stable critical points are local minima of $H$.

Proof: The proof of this theorem follows readily from Lemma 1 , using the fact that $H$ is locally Lipschitz and regular, and that there exists a compact and strongly invariant set for (1). The maximum of a finite set of continuously differentiable functions is a locally Lipschitz and regular function [3]. Thus the function $f_{i j}$ is regular because it can be written as $f_{i j}=\max \left\{\frac{P_{0}}{d_{i j}^{\alpha}+1}-C, 0\right\}$ where both $f\left(d_{i j}\right)=\frac{P_{0}}{\alpha_{i j}^{\alpha}+1}-C$ and $f\left(d_{i j}\right)=0$ are continuously differentiable functions and thus $f_{i j}$ is a locally Lipschitz and regular function. Combining equations (3) and (2), it is clear that $H$,from (4), is an algebraic composition of signal strength functions. Since the signal-strength function $f_{i j}$ is Lipschitz and regular, by applying the Sum Rule and Quotient Rule it follows that $\mathrm{H}$ is both Lipschitz and Regular. Lastly, we show that there exists a compact and strongly invariant set for the dynamical system in (1). The generalized gradient $\frac{\partial H}{\partial x_{i}}$ for agent $i$ goes to zero when agent $i$ is outside of the communication radius $R$ for all other $N-1$ agents and thus we define the set, $\mathcal{M}$, to be the set of points for which the generalized gradient is non-zero. Let $\mathcal{M} \subseteq \mathbb{R}^{d}$ be the set of all points inside the radius $2 R(N-1)$ from the origin where, for the case of one ground robot $g$, we place $g$ at the origin. By definition this set is both closed and bounded in a ball $B(0,2 R(N-1))$ and is thus compact. This generalizes readily to the case of more than one ground robot if we find the union of all such sets. Furthermore, a solution to (1) with any initial condition $x_{0} \in \mathcal{M}$ remains in $\mathcal{M}$ because $\frac{\partial H}{\partial x_{i}}(p)=0 \forall p \notin \mathcal{M}$ and so $\mathcal{M}$ is a strongly invariant set.

Using the Product Rule and the Sum Rule, and the fact that $f_{i j}$ is Lipschitz and regular, we now present the final form of our controller from (1). 


$$
\begin{aligned}
& \dot{x}_{i}=-\operatorname{Ln}\left[\frac{\partial H}{\partial x_{i}}\right] \\
& =\sum_{i=1}^{N} \sum_{j=1}^{N}-\frac{\partial S I R_{i j}}{\partial x_{i}}\left(1+\lambda\left(S I R_{i j}+\delta\right)^{-2}\right) .
\end{aligned}
$$

Where $\frac{\partial f_{i j}}{\partial x_{i}}$ was defined above in (6) and $\frac{\partial S I R_{i j}}{\partial x_{i}}$ is

$$
\begin{aligned}
& \frac{\partial S I R_{i j}}{\partial x_{i}}= \\
& \frac{\frac{\partial f_{i j}}{\partial x_{i}}}{N_{i}+\sum_{k \in \mathcal{N}_{i} \backslash j} f_{i k}}-f_{i j} \frac{\frac{\partial N_{i}}{\partial x_{i}}+\sum_{k \in \mathcal{N}_{i} \backslash j} \frac{\partial f_{i k}}{\partial x_{i}}}{\left(N_{i}+\sum_{k \in \mathcal{N}_{i} \backslash j} f_{i k}\right)^{-2}}
\end{aligned}
$$

\section{B. Connectivity Maintenence}

We use the fact that the aerial vehicles are following a gradient descent on the cost function $H$ to identify initial conditions that prevent agents from moving to disconnect the communication graph. Because of the distributed nature of our controller, we do not employ any global checks on graph connectivity and thus require that the communication graph is initially connected. We present two approaches to maintaining graph connectivity. The first approach identifies the minimum cost of a disconnected network and requires that the initial conditions of any network are below this value. The second approach is to find a critical value of $\lambda$ in (4) such that aerial vehicles will never move outside of a radius $R$ from their neighbors and thus will remain connected. The main difference between these two approaches is that the first approach is a check on initial conditions to ensure that connectivity is maintained, while the second approach is a design perspective where a value of the parameter $\lambda$ is chosen as a function of other parameters in (4) to prevent disconnection.

Theorem 2: Given that the network begins in a connected state, the aerial vehicles will not move in such a way to disconnect the graph under either of the two following conditions:

1) The initial cost of the system $H$ begins below the minimum cost of a disconnected graph $H_{d_{\min }}$.

2) The design parameter, $\lambda$, in (4) takes a value $\lambda \geq \lambda_{\text {crit }}$ where $\lambda_{\text {crit }}$ is the value at which the dot product $\frac{\partial H^{T}}{\partial x_{i}}\left(x_{i}-x_{j}\right)=0$ for the pair $i-j$ where $d_{i j}^{*}=\max \left\|x_{i}-x_{j}\right\|$ s.t. $d_{i j}^{*}<R$.

Proof: We identify the minimum cost of a disconnected graph that we call $H_{d_{\min }}$. Because our controller requires that agents will move to decrease the cost, $H$, if the initial cost of the system $H_{0}<H_{d_{\min }}$ then the network will remain connected. For the second part of the theorem we identify a value of the parameter $\lambda$ such that an agent will never disconnect from its neighbors in the worst-case scenario. Namely, we ensure that the dot product ${\frac{\partial H}{\partial x_{i}}}^{T}\left(x_{i}-x_{j}\right)=$ 0 in the limit as $d_{i j} \rightarrow R$ so that agent $i$ 's velocity component in the direction away from $j$ is zero and thus will never disconnect an existing connection. This is depicted graphically in Figure 3 .

1) Minimum cost of a Disconnected Network: The cost of disconnecting an edge in the communication graph, or equally, the cost of a missing connection in the communication graph is given by:

$$
\left.H_{i j}\right|_{d_{i j}=R}=\frac{\lambda}{\delta}
$$

To find the minimum cost of a disconnected graph, we find the minimum number of missing connections for a disconnected graph. If we look at the case of two disconnected subgraphs, the number of elements in each subgraph is $s$ and $N-s$ respectively, where $N$ is the total number of elements. The function $c(s)=s(N-s)$ denotes the number of missing connections between the two subgraphs (we assume subgraphs are fully connected). Minimizing $c(s)$ w.r.t. $k$ yields $s=1$, meaning that the minimum number of disconnections in a graph is acheived when $s=1$. All other cases where the number of subgraphs is less than one is a subcase of this one. Therefore we find that the minimum number of edge disconnections for a disconnected graph is $2(N-1)$ and the cost for this graph is:

$H_{d}=2(N-1) \frac{\lambda}{\delta}+\sum_{u \neq s} \sum_{w \neq s}-S I R_{u w}+\lambda\left(S I R_{u w}+\delta\right)^{-1}$

Furthermore, we are interested in the minimum cost of such a graph. The theoretical minimum of Equation (4) would be acheived when the SIR value for all the agents in the second subgraph is maximal. The maximum theoretical value of the $S I R_{i j}$ from Equation (2) is acheived when the distance of the two agents $i$ and $j$ goes to zero and when interfering communication from $i$ 's neighbors, or environmental noise $N_{i}$ is not accounted for. This maximum is the same maximum as that of $f_{i j}$ and is $\max \left\{S I R_{i j}\right\}=P_{0}-C$. Plugging this into the cost function we find the minimum possible $H$ for a disconnected graph:

$$
\begin{aligned}
H_{d_{\min }}= & 2(N-1) \frac{\lambda}{\delta}- \\
& (N-1)(N-2)\left(\left(P_{0}-C\right)-\lambda\left(\left(P_{0}-C\right)+\delta\right)^{-1}\right)
\end{aligned}
$$

Therefore we conclude that if the initial configuration has a cost $H_{\text {initial }}<H_{d_{\text {min }}}$ then the aerial vehicles will remain connected for all time.

2) Finding Critical Value of $\lambda$ to Ensure Connectivity: We find the $\lambda$ for which two agents that are currently neighbors, will not move a distance larger than $R$ from each other. The intuition behind this critical $\lambda$ value is the observation that as the distance between two agents $i-j$ approaches the communication radius $R, \lambda$ can be chosen such that the generalized gradient $\frac{\partial H}{\partial x_{i}}$ will have a zero component in the direction pointing away from $j$, and thus the agent $i$ 


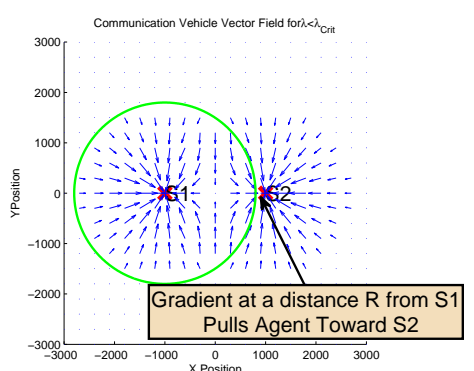

(a)

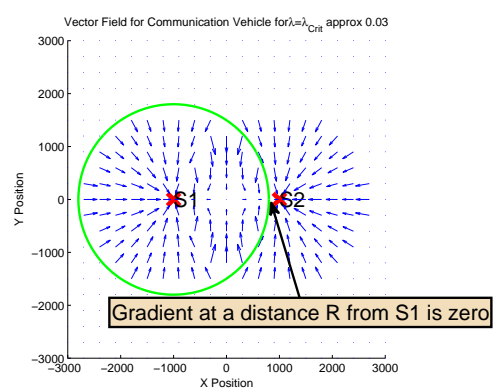

(b)

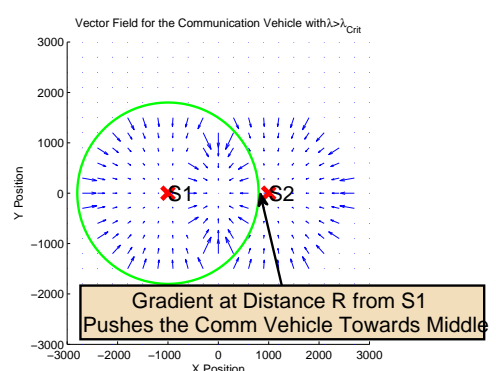

(c)

Fig. 3. This plot shows the force felt by a communication vehicle in the presence of two ground (sensor) agents, S1 and S2. It demonstrates the effect of the design parameter $\lambda$ on the communication vehicle gradient field where connectivity is maintained for $\lambda \geq \lambda_{\text {Crit }}$. Figures a through $\mathrm{c}$ show how the controller exhibits greedy SIR-maximizing behavior for small $\lambda$ values and an increasingly symmetric configuration demonstrating a balanced SIR over all links for larger $\lambda$ values.

will never move further than the distance $R$ away from $j$, $\forall j \in \mathcal{N}_{i}$. This corresponds to the $\lambda$ that forces

$$
-\frac{\partial H^{T}}{\partial x_{i}}\left(x_{i}-x_{j}\right)=0
$$

Where the vector $\left(x_{i}-x_{j}\right)$ points from $j$ to $i$ and $j$ is a neighbor at a distance approaching $R$ from $i$. We expand Equation (13):

$$
\begin{aligned}
& -\left(\frac{\partial H_{i j}}{\partial x_{i}}+\frac{\partial H_{j i}}{\partial x_{i}}+\sum_{\{u, w\} \neq\{i, j\},\{j, i\}} \frac{\partial H_{u w}}{\partial x_{i}}\right)^{T} \\
& *\left(x_{i}-x_{j}\right)=0
\end{aligned}
$$

Where

$$
\frac{\partial H_{u w}}{\partial x_{i}}=-\frac{\partial S I R_{u w}}{\partial x_{i}}\left(1+\lambda\left(S I R_{u w}+\delta\right)^{-2}\right)
$$

As seen in Equation (14) and (15), the gradient-based controller for agent $i$ is a combination of the gradients of the SIR values between $i$ and $k, \forall k \in \mathcal{N}_{i}$, weighted by the inverse of the value of the SIR for that pair $x_{i}-x_{k}$. This weighting is directly influenced by $\lambda$, but goes to zero when $\lambda=0$. Therefore, it is intuitive that a larger $\lambda$ value will amplify the effect of the value $S I R_{u w} \rightarrow 0$ in Eq (15), and thus the contribution of the gradient on $i$ from the agent whose distance is approaching $R$ will dominate for larger values of $\lambda$. Solving for $\lambda$ from Equation (14), we find:

$$
\lambda=\frac{-\sum_{u}^{N} \sum_{w}^{N} \frac{\partial S I R_{u w}}{\partial x_{i}}{ }^{T}\left(x_{i}-x_{j}\right)}{\sum_{u}^{N} \sum_{w}^{N}\left(S I R_{u w}+\delta\right)^{-2}{\frac{\partial S I R_{u w}}{\partial x_{i}}}^{T}\left(x_{i}-x_{j}\right)}
$$

As the distance $d_{i j} \rightarrow R$, we note that:

$$
\begin{aligned}
& \frac{\partial S I R_{i j}{ }^{T}}{\partial x_{i}}\left(x_{i}-x_{j}\right) \rightarrow \\
& \quad \alpha P_{0}\left(R^{\alpha}+1\right)^{-2} R^{\alpha-2}\left(N i+\sum_{k \in \mathcal{N}_{i}} f_{i k}\right)^{-1} R^{2} .
\end{aligned}
$$

and

$$
S I R_{i j}=S I R_{j i} \rightarrow \frac{1}{\delta}
$$

To find $\lambda_{\text {crit }}$ we must analyze the upper bound to the equation (16). This corresponds to finding the case where the link $i-j$ is most easily disconnected. From the Equation (14) we see that the upper bound is when the gradient dot product $\frac{\partial H_{u w}}{\partial x_{i}}\left(x_{i}-x_{j}\right)$ is maximized, or equivalently, when all agents $x_{k} \neq x_{j}$ have a maximum value of the gradient $\frac{\partial H_{u w}}{\partial x_{i}}$ in the direction exactly opposite to the vector $\left(x_{i}-x_{j}\right)$. If we ignore agent interference in the Signal-to-Interference Ratio to get a upper bound on $H_{u w}$, this is the case where all agents not including $j$ are co-located at a point that is opposite of the direction $i-j$ with respect to $i$ so that the vector exactly opposite to $\left(x_{i}-x_{j}\right)$ is $\left(x_{w}-x_{i}\right)$. We place all $N-2$ agents at a distance $R-\gamma$ from $i$, where

$$
\gamma=\arg \max _{\gamma} \frac{\partial H_{w i}{ }^{T}}{\partial x_{i}}\left(x_{w}-x_{i}\right)
$$

Thus the smallest value of lambda for which we are guaranteed to preserve connectivity is:

$$
\begin{aligned}
& \lambda_{\text {crit }}=-\left(-\frac{\alpha P R^{\alpha}}{\left(R^{\alpha}+1\right)^{2}\left(N_{i}+(N-2) P\right)}-\frac{\alpha P R^{\alpha}}{\left(R^{\alpha}+1\right)^{-2} N_{j}}\right.
\end{aligned}
$$

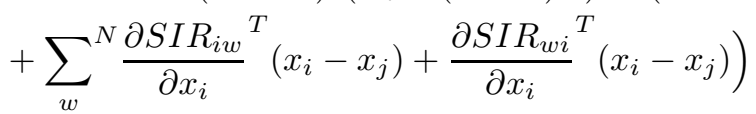

$$
\begin{aligned}
& *\left(2\left(\frac{1}{\delta}\right)^{2}+\sum_{w}^{N}\left(S I R_{i w}+\delta\right)^{-2}{\frac{\partial S I R_{i w}}{\partial x_{i}}}^{T}\left(x_{i}-x_{j}\right)\right. \\
& \left.+\left(S I R_{w i}+\delta\right)^{-2}{\frac{\partial S I R_{w i}}{\partial x_{i}}}^{T}\left(x_{i}-x_{j}\right)\right)^{-1}
\end{aligned}
$$

Placing all neighbors $k$, not including $j$, of $i$ at a distance $(R-\gamma)$ from $i$, and using the upper bound on SIR by ignoring all third party neighbor interference in the SIR terms except interference from $j$, we find the following expressions which can be plugged into the above equation to find $\lambda_{\text {crit }}$ :

$$
\begin{aligned}
& {\frac{\partial S I R_{i w}}{\partial x_{i}}}^{T}\left(x_{i}-x_{j}\right)=-\frac{a_{i w}}{N_{i}}(R-\gamma) R- \\
& \frac{\frac{P_{0}}{(R-\gamma)^{\alpha}}-C}{N_{i}^{2}}\left(\frac{\partial N_{i}}{\partial x_{i}}\left(x_{i}-x_{j}\right)+a_{i j} R^{2}\right)
\end{aligned}
$$


and

$$
\begin{aligned}
& {\frac{\partial S I R_{w i}}{\partial x_{i}}}^{T}\left(x_{i}-x_{j}\right)=-\frac{a_{w i}}{N_{w}}(R-\gamma) R \\
& S I R_{i w}=\left(\frac{P_{0}}{(R-\gamma)^{\alpha}+1}-C\right)\left(N_{i}\right)^{-1} \\
& S I R_{w i}=\left(\frac{P_{0}}{(R-\gamma)^{\alpha}+1}-C\right)\left(N_{w}\right)^{-1} \\
& a_{i w}=a_{w i}=\alpha P_{0}\left((R-\gamma)^{\alpha}+1\right)^{-2}(R-\gamma)^{\alpha-2}
\end{aligned}
$$

Because we have found the minimum value of $\lambda$ for which $-{\frac{\partial H}{\partial x_{i}}}^{T}\left(x_{i}-x_{j}\right)=0, \forall j$, we have shown that if we choose $\lambda \geq \lambda_{\text {crit }}$, agent $x_{i}$ will never move out of the ball of radius $R$ centered at $x_{j}$.

\section{RESUlTS}

In this section we present the results of implementing our controller on a quadrotor hardware testbed, hardware-in-theloop simulations, and MATLAB simulations.

\section{A. Hardware Implementation}

We tested our controller on a group of three aerial vehicles which are AscTec Hummingbird flying quad-rotor robots each with an ARM micro-processor and $2.4 \mathrm{GHz}$ xBee modules for wireless communication, and three ground vehicles. We conducted the experiments in a room equipped with a Vicon motion capture system where position information was broadcasted wirelessly to each robot and all computation was performed onboard each of the robots in real time. For our hardware experiments we set the controller parameters $\lambda=1>\lambda_{\text {crit }}$ and $\delta=0.001$, and the communication parameter $\alpha=2$. We demonstrate that the aerial vehicles acheive a configuration that locally minimizes the cost $H$. Figure 4 shows minimization of the cost function $H$ averaged over ten trials with errorbars indicating the one standard deviation around the mean. Each experiment lasted on the order of one minute.

We demonstrate the adaptive capabilities of the controller by disabling one of the aerial vehicles and relocating this aerial vehicle to a fixed position on the ground. As shown in Figure 4, the remaining aerial vehicles re-adjust their equilibrium position to compensate for this change in the system. Figure 4

\section{B. Hardware-in-the-Loop Simulation}

We tested our controller on a total of 7 ARM microcontrollers communicating wirelessly via $\mathrm{xBee}$-XSC wireless modules. The tests were conducted on four ground vehicles, and three aerial communication vehicles with control parameters $\lambda=1>\lambda_{\text {crit }}$ and $\delta=0.001$. Figure 5 shows the minimization of the cost and Figure the trajectories of the aerial vehiles with final equilibrium positions marked as blue circles.

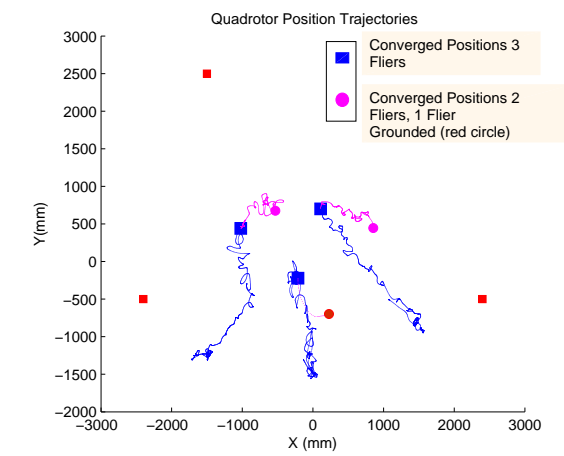

(a) Initial and Converged Positions for Hardware Trial.

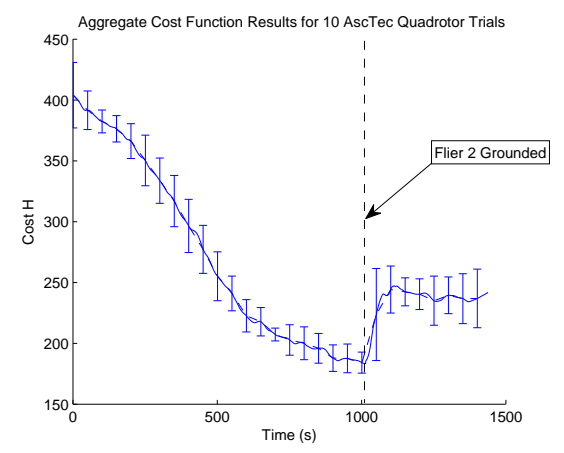

(b) Aggregate Cost Function over Ten Hardware Trials.

Fig. 4. Position trajectories and aggregate cost function for three fliers (shown as blue solid line in Fig 6(a) ) with flier equilibrium positions marked as blue squares and ground vehicle positions marked as red squares. After reaching equilibrium one of the fliers is deactivated and moved to the side while the remaining fliers find a new equilibrium position (postdeactivation trajectories shown in dotted magenta line).

\section{MATLAB Simulation}

We test a configuration with 16 total vehicles, where 8 are ground sensors and the remaining 8 are aerial communication vehicles. We set the control parameters $\delta=0.001$ and the $\lambda$ parameter to $\lambda=10>\lambda_{\text {crit }}$ to target equalized SIR values amongst aerial vehicles. The aerial vehicles shown in blue have initial positions at a depot in the top right and bottom left corners. Green circles denote the communication radius of the farthest sensors, sensors 1 and 6, to demonstrate that aerial vehicles are initialized out of communication range with other sensors and aerial vehicles in the team. The resulting agent trajectories and cost function demonstrates non-smooth transitions for the points where agents enter each others communication radius as shown in Figure 6 .

\section{CONCLUSion}

This paper presents the formulation of a distributed controller to optimize signal-link quality amongst a team of air and ground vehicles, where the ground vehicles are performing a collaborative task independent of the aerial vehicles, and the task of the air vehicles is to position themselves to optimize signal-quality amongst all vehicles in the network. We control the aerial vehicles via gradient descent on a cost function comprised of a continuous, physically-based 


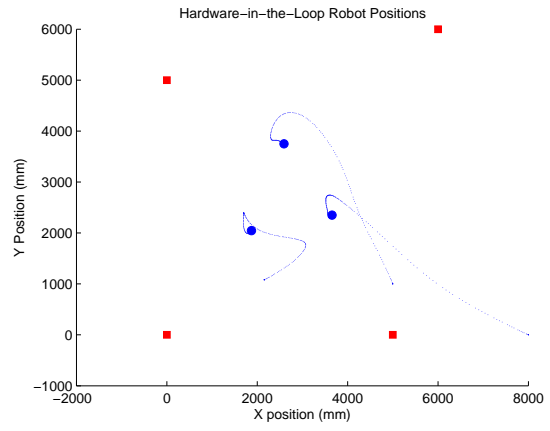

(a) Hardware-in-the-Loop Initial and Converged Positions.

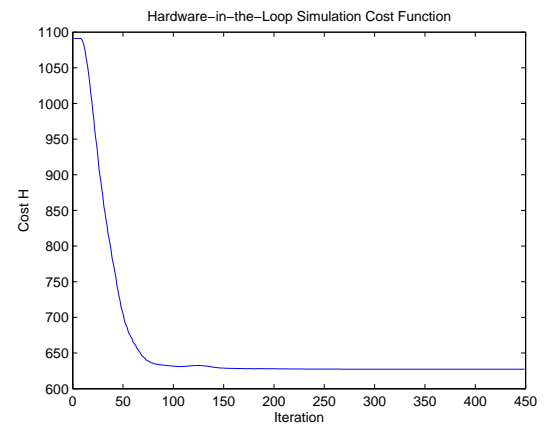

(b) Hardware-in-the-Loop Cost Function.

Fig. 5. Position data and cost function for hardware-in-the-loop simulation where aerial vehicle trajectories are shown as blue lines and converged positions as blue dots. The ground vehicles are plotted as red squares in this figure.

measure of signal quality, the Signal-to-Interference Ratio. We assume that agents are only in communication within a radius $R$ and our provably convergent controller allows for neighbors to enter and exit each other's communication neighborhood in a nonsmooth manner. We demonstrate our controller in hardware experiments using AscTech quad-rotor vehicles, in hardware-in-the-loop simulations, and in MATLAB simulations, demonstrating the positioning of the aerial vehicles to minimize the cost function $H$ and improve signalquality amongst all communication links in the ground/air robot team.

\section{Acknowledgements}

The authors would like to thank Wil Selby and Lauren White for their help with hardware experiments.

\section{REFERENCES}

[1] W. Truszkowski, M. Hinchey, J. Rash, and C. Rouff, "Nasa's swarm missions: the challenge of building autonomous software," IT Professional, vol. 6, pp. 47-52, 2004.

[2] P. Gupta and P. R. Kumar, "The capacity of wireless networks," 1999.

[3] J. Cortes, "Discontinuous dynamical systems," Control Systems Magazine,IEEE, vol. 28, pp. 36-73, 2008.

[4] M. Pavone, E. Frazzoli, and F. Bullo, "Distributed policies for equitable partioning: Theory and applications," in Decision and Control, IEEE International Conference on, 2008.

[5] E. Frazzoli and F. Bullo, "Decentralized algorithms for vehicle routing in a stochastic time-varying environment," in Decision and Control, IEEE International Conference on, 2004.

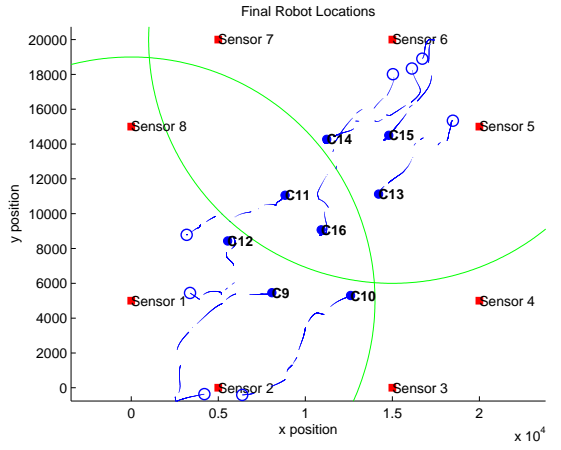

(a) Initial and Converged Positions.

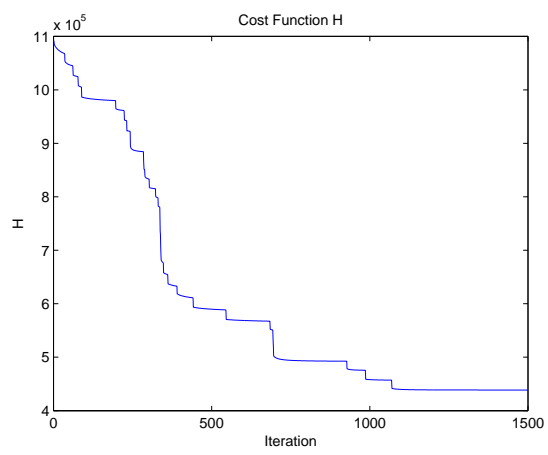

(b) Non-smooth Cost Function.

Fig. 6. Matlab simulation results of converged positions and position trajectories for 8 aerial vehicles and 8 ground vehicles with non-smooth cost function $\mathrm{H}$. Initial aerial vehicle positions are shown as blue circles, converged positions are shown as filled blue circles, and trajectories are shown as a blue line in Figure 6(a). Communication radius of senors 1 and 6 shown in green demonstrate that not all agents are in communication initially. Trajectories as well as cost function show non-smooth transitions at the points where agents enter each others communication neighborhood.

[6] S.-K. Yun and D. Rus, "Optimal distributed planning of multi-robot placement on a 3d truss," in Intelligent Robots and Systems,Proc of IEEE International Conference on, 2007.

[7] M. Schwager, B. Julian, and D. Rus, "Optimal coverage for multiple hovering robots with downward-facing cameras," in Robotics and Automation, Proc of International Conference on, 2009.

[8] J. Cortes, S. Martinez, T. Karatas, and F. Bullo, "Coverage control for mobile sensing networks," in IEEE Transactions of Robotics and Automation, 2004.

[9] N. Ayanian, V. Kumar, and D. Koditschek, "Synthesis of controllers to create, maintain, and reconfigure robot formations with communication constraints," in Intelligent Robotic Systems, IEEE International Conference on, 2009.

[10] E. Stump, A. Jadbabaie, and V. Kumar, "Connectivity management in mobile robot teams," in Robotics and Automation, IEEE International Conference on, 2008, pp. 1525-1530.

[11] O. Burdakov, P. Doherty, K. Holmberg, J. Kvarnstrom, and P. R. Olsson, "Positioning unmanned aerial vehicles as communication relays for surveillance tasks," in Robotics Science and Systems, Conference on, 2009.

[12] E. W. Frew, "Information-theoretic integration of sensing and communication for active robot networks," Mobile Networks and Applications, vol. 14, pp. 267-280, 2009.

[13] N. Michael, M. Zavlanos, V. Kumar, and G. Pappas, "Maintaining connectivity in mobile robot networks," in Experimental Robotics, ser. Springer Tracts in Advanced Robotics. Springer Berlin/Heidelberg, 2009, vol. 54, pp. 117-126.

[14] A. Cornejo, R. Ley-Wild, F. Kuhn, and N. Lynch, "Keeping mobile robot swarms connected," MIT-CSAIL, Tech. Rep., June 2009. 